\title{
Treatment of angina pectoris with nifedipine and atenolol: efficacy and effect on cardiac function
}

\author{
IAIN N FINDLAY, KAY MACLEOD, MARTIN FORD, GERARD GILLEN, \\ ALEX T ELLIOTT, HENRY J DARGIE \\ From the Department of Cardiology, Western Infirmary, Glasgow; and Department of Medical Physics and \\ Bio-engineering, Glasgow
}

SUMMARY The antianginal effects of nifedipine $20 \mathrm{mg}$ three times a day and atenolol $100 \mathrm{mg}$ once a day singly and in combination were investigated in 16 patients with angina pectoris. The amount of work that could be done before angina and ST depression appeared was significantly increased by atenolol and the combination but not by nifedipine. At peak exercise the number of leads on a 16 point precordial electrocardiogram map that demonstrated $\geqslant 1 \mathrm{~mm} S T$ segment depression was significantly reduced from a mean (SD) of $5.0(0.4)$ on placebo to $3.7(0.6)$, $2 \cdot 8(0 \cdot 4)$, and $2 \cdot 3(0 \cdot 7)$ on nifedipine, atenolol, and the combination respectively. Mean resting left ventricular ejection fraction, assessed by gated radionuclide ventriculography, did not change during any active treatment phase but increased significantly during exercise only on nifedipine and the combination.

The nifedipine/atenolol combination was the most effective treatment, and the data suggest that nifedipine may be used to best advantage in combination with a beta blocker.

Despite the introduction of calcium antagonists, beta adrenoceptor blockers remain the standard treatment for chronic stable angina pectoris in the United Kingdom. But the differing and possible complementary actions of beta blockers and calcium antagonists suggest the possibility of a beneficial drug interaction; indeed several studies of the combination of nifedipine or verapamil and beta blockers indicate an additive effect. ${ }^{1-3}$

Concern has been expressed about the possible depressant effects of this combination on cardiac function, however, ${ }^{4-6}$ and, theoretically, reduction of sympathetic stimulation by beta blockade together with the intrinsic negative inotropic effect of calcium antagonism could depress myocardial contractility despite the peripheral vasodilating effect of the latter.

We have investigated the antianginal effects of a calcium antagonist (nifedipine) and a beta blocker (atenolol) singly and in combination on symptoms, exercise induced ischaemia, and left ventricular function.

Requests for reprints to $\mathrm{Dr} \mathrm{H}$ J Dargie, Western Infirmary, Glasgow G11 6NT.

\section{Patients and methods}

\section{PATIENTS}

We recruited sixteen male patients from the cardiology clinic of the Western Infirmary, all of whom had had chronic effort related angina pectoris with a stable pattern of symptoms for at least 3 months; none had pain at rest. The mean (SE) age of the group was 56(2) years (range 45-63). All had an unequivocally positive exercise test for ischaemia $(\geqslant 0.1 \mathrm{mV} \mathrm{ST}$ segment depression for $80 \mathrm{~ms}$ after the $J$ point) at the end of a two week placebo run-in period, during which all other antianginal medication had been withdrawn. Coronary arteriography showed coronary artery disease in all patients; 10 had triple, three had double, and three had single vessel disease. Eight had had a previous myocardial infarction and six patients were cigarette smokers who did not change their habit during the study period.

Exclusion criteria included chronic obstructive airways disease, blood pressure $>160 / 100 \mathrm{~mm} \mathrm{Hg}$ on no treatment, myocardial infarction within the previous 3 months, peripheral vascular disease, and previous congestive cardiac failure or pronounced conduction abnormalities on the electrocardiogram. 
Written consent was obtained from all patients and the study was approved by the ethical committee of the Western Infirmary.

\section{TRIAL DESIGN}

There were four treatment periods each lasting 3 weeks and comprising placebo, nifedipine $20 \mathrm{mg}$ three times a day, and atenolol $100 \mathrm{mg}$ once daily singly and that combination. The order of administration was determined by a balanced latin square, and use of the double dummy technique ensured that all treatments were visually identical.

\section{SUBJECTIVE ASSESSMENT}

Patients completed diary cards for anginal frequency and glyceryl trinitrate consumption and they were instructed to follow their normal daily routine throughout the period of the trial; moreover patients were instructed to use glyceryl trinitrate to treat acute attacks of angina (but not dyspnoea) and were asked not to take it prophylactically.

\section{OBJECTIVE ASSESSMENT}

Supine bicycle exercise was carried out on two separate occasions, to record an exercise precordial map and to assess cardiac function by gated radionuclide ventriculography.

\section{EXERCISE PRECORDIAL MAPPING}

Exercise was performed on an electronically braked bicycle ergometer, and was started at $25 \mathrm{~W}$. The workload was increased by $25 \mathrm{~W}$ every 3 minutes. Each patient was allowed to exercise to his maximum; the end points were severe chest pain, dyspnoea, intolerable fatigue, important arrhythmia, or fall in blood pressure. Immediately after exercise, ST segment change was recorded on a 16 point precordial electrocardiograph map. ${ }^{7}$ Heart rate and blood pressure were recorded throughout the test.

\section{RADIONUCLIDE VENTRICULOGRAPHY}

The blood pool was visualised by in vivo labelling of the patient's red blood cells with $800 \mathrm{mBq}$ of technetium- $99 \mathrm{~m}$. Scintigraphic images were obtained with a single crystal gamma camera (Ohio series 100) fitted with a high resolution parallel hole collimater interfaced to a Varian computer. Patients were studied while they were supine and imaging was carried out in the left anterior oblique projection at an angle that allowed optimum separation of the right and left ventricles and showed minimal interventricular septal thickness (generally $35-45^{\circ}$ left anterior oblique); a $10-15^{\circ}$ caudal tilt was applied to allow image separation of the left atrium from the left ventricle and this position was kept constant for each patient throughout the study.
Electrocardiogram gated equilibrium data were collected at rest for three minutes and during the last two minutes of supine bicycle exercise at the maximum workload achieved during the placebo run-in period. The global left ventricular ejection fraction was calculated from the standard formula: end diastolic counts - end systolic counts/end diastolic counts-background counts using a peridiastolic background area along the left ventricular free,wall.

Statistical analysis was performed by Student's paired $t$ test unless stated otherwise.

\section{Results}

\section{SUBJECTIVE ASSESSMENT}

When compared with placebo results the frequency of angina was reduced significantly by nifedipine $(p<0.05)$ and atenolol $(p<0.02)$ singly and in combination $(p<0.005)$. Glyceryl trinitrate consumption was reduced by atenolol $(p<0.02)$ and the combination $(p<0.01)$, but during the nifedipine period this did not reach statistical significance (Fig. 1).

\section{OBJECTIVE ASSESSMENT}

\section{Exercise precordial map}

There was no significant increase in total work done during treatment with nifedipine or atenolol when compared with placebo; only the combination significantly increased the total work done (mean $(\mathrm{SE}))$ from $44(6)$ to $51(7) \mathrm{kJ}(\mathrm{p}<0.05)$. Both atenolol and the combination significantly increased the work done to the onset of angina and to the onset of ST segment depression $(p<0.05$ and $p<0.02$ for atenolol and $p<0.005$ and $p<0.02$ for the combination respectively, by log rank analysis). ${ }^{8}$ All active treatments reduced both the frequency of angina during exercise and the development of ST segment depression (Fig. 2). Angina pectoris was the limiting symptom in 12 , eight, three, and one of the patients on placebo, nifedipine, atenolol, and the combination respectively.

Immediately after exercise the mean (SE) number of leads showing $>1 \mathrm{~mm} \mathrm{ST}$ segment depression was reduced from $5.0(0.4)$ on placebo to $3.68(0.6)$ $(p<0.005), 2.81(0.4) \quad(p<0.005)$, and $2.25(0.7)$ $(\mathrm{p}<0.005)$ on nifedipine, atenolol, and the combination respectively. The mean (SE) total ST segment depression $(\mathrm{mm})$ was reduced from $8.75(1.5)$ on placebo to $7.0(1.9)$ (NS), $4.2(1)(\mathrm{p}<0.001)$, and $3.87(1.5)(p<0.005)$ on nifedipine, atenolol, and the combination respectively.

Resting heart rate (mean (SE)) did not change on nifedipine $(68(2)$ to $71(2)$ beats per minute), whereas both atenolol and the combination lowered resting heart rate significantly and to the same extent 


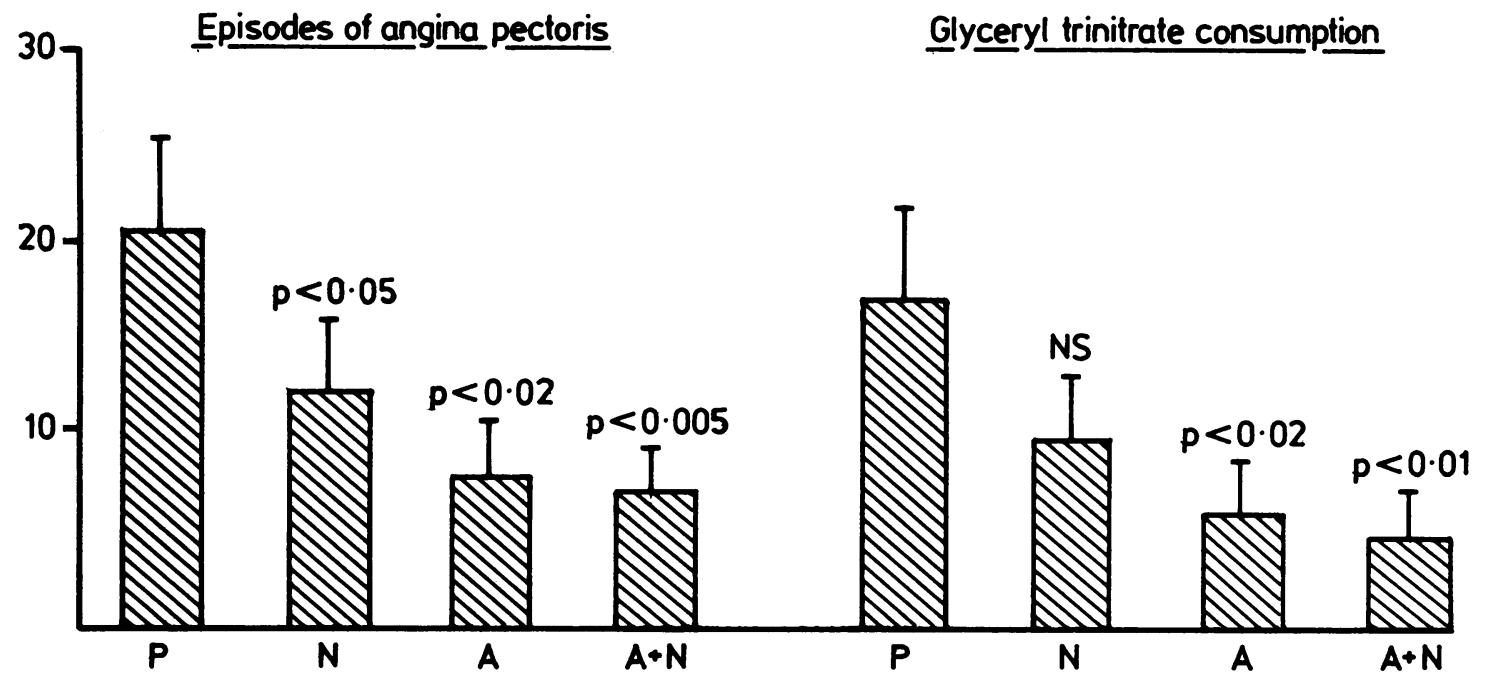

Fig. 1 Frequency of angina pectoris and glyceryl trinitrate consumption during each three week treatment period; values are mean (SE). P, placebo; $N$, nifedipine; $A$, atenolol; $A+N$, combination.

(54(2) and $54(2)$ beats per minute, $p<0.001)$. During exercise there was no significant difference in the peak rate pressure product (mean (SE)) on nifedipine and placebo $(230(13)$ vs $234(15) \times 100)$ but there was a highly significant reduction on atenolol and the combination $(177(8)$ and $174(11) \times 100$, $\mathrm{p}<0.001)$.

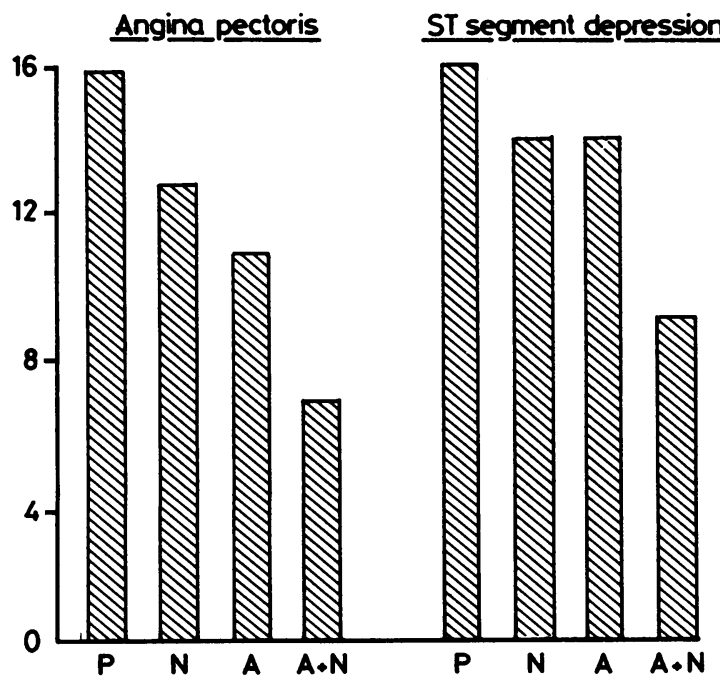

Fig. 2 Number of patients developing angina pectoris or $S T$ segment depression at maximum exercise. See legend to Fig. 1 for abbreviations.
There were no deleterious effects on cardiac rhythm or conduction during any treatment phase.

\section{RADIONUCLIDE STUDIES}

Mean resting ejection fraction did not change significantly on any of the active treatment phases (Table). But when individual values were examined there was a correlation between the change in ejection fraction on combination treatment and the resting value on placebo (Fig. 3); in those patients with higher resting ejection fraction there was a fall, while in those with lower resting placebo values there was a slight rise; this was not evident when nifedipine or atenolol was given alone. During the exercise scan 14,11 , three, and one patients developed angina on placebo, nifedipine, atenolol, and the combination respectively; during both nifedipine and combination treatment there were statistically significant rises in left ventricular ejection fraction on exercise (Table).

The normal response of left ventricular ejection fraction to maximum dynamic exercise in our laboratory is a rise $\geqslant 7 \%$. On placebo four $(25 \%)$ patients had a normal response despite the presence of angina in three, while on nifedipine eight $(50 \%)$ had a normal response despite the presence of angina in six; on atenolol only one $(6 \%)$ patient had a normal rise in left ventricular ejection fraction despite 13 of the 16 patients being free of angina; on the combination six $(40 \%)$ patients had a normal rise in left ventricular ejection fraction and only one patient developed angina (Table). 
Table Left ventricular ejection fraction ( $L V E F$ ) response to dynamic exercise in the presence or absence of angina pectoris at time of acquisition. Mean (SE)

\begin{tabular}{lllll}
\hline & Placebo & Nifedipine & Atenolol & Combination \\
\hline Resting LVEF & $60(2)$ & $60(2)$ & $60(2)$ & $58(1)$ \\
Exercise LVEF & $63(2)$ & $65(3)$ & $61(2)$ & $62(2)$ \\
Angina pectoris during exercise & 14 & $\mathrm{p}<0.02$ & 3 & $\mathrm{p}<0.005$ \\
Normal exercise LVEF & 4 & 8 & 1 & 6 \\
Normal exercise LVEF despite angina & 3 & 6 & 1 & 1 \\
Normal exercise LVEF without angina & 1 & 2 & 0 & 5 \\
\hline
\end{tabular}

\section{Discussion}

In a previous double blind placebo controlled study of identical design nifedipine enhanced the antianginal effect of propranolol but when given alone it was less effective than the $\beta$ blocker. ${ }^{1}$ In general the present study confirms these findings for both nifedipine and atenolol given singly or in combination. Although the mode of action of $\beta$ blockers in alleviating myocardial ischaemia by blunting the heart rate and blood pressure responses to exercise seems clear, the mechanism of action of nifedipine in exercise induced ischaemia is less obvious. Principally nifedipine is a vasodilator and it could act either by increasing coronary blood flow or by reducing myocardial oxygen consumption through its favourable effect on left ventricular afterload. ${ }^{9-11}$ In a clinical trial these effects are difficult to separate and the balance of effects on the coronary and peripheral circulations cannot be determined with certainty. If an increase in coronary blood flow had occurred in our patients then the severity of ischaemia at any given rate pressure product would have been less. Nifedipine did not prolong exercise duration or

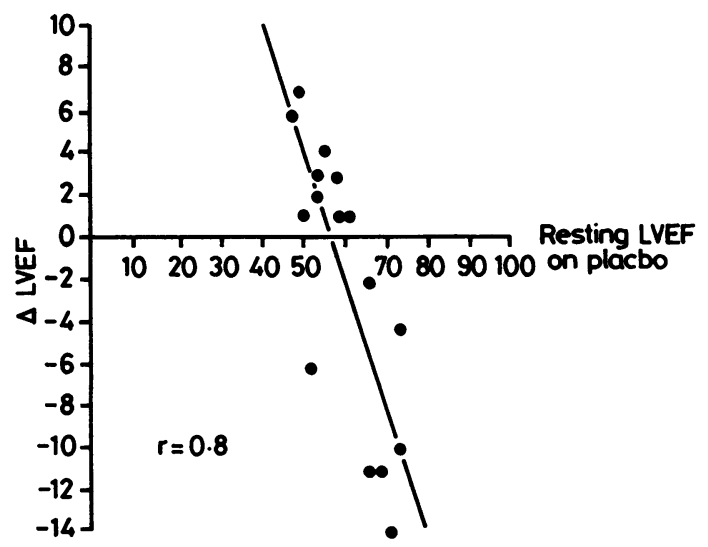

Fig. 3 Change in resting left ventricular ejection fraction ( $\triangle L V E F$ ) during combination therapy. $\triangle L V E F$ equals result on combination minus result on placebo. reduce the rate pressure product when compared with placebo but there was a modest reduction in the severity of ST segment depression at maximum exercise. Thus, these data do not exclude a small beneficial effect on coronary blood flow. But in patients with atheromatous coronary artery disease and effort related angina the most important antianginal effect may be reduction of myocardial oxygen consumption in relation to total external work done. It is not surprising therefore that atenolol, which considerably reduced the rate pressure product, had greater effects on exercise induced ischaemia than did nifedipine.

Although hypotension and left ventricular failure have been described in anecdotal reports, ${ }^{12-14}$ objective studies in which nifedipine was given to patients already established on a $\beta$ blocker have been reassuring. ${ }^{15-17}$

The principal aim of the present study was to investigate in a double blind study the long term effects of this combination in patients with unimpaired left ventricular function before treatment. We assessed this by radionuclide ventriculography both at rest and during exercise and we have shown that at rest there was no significant change in the mean left ventricular ejection fraction during any treatment period. The normal response of left ventricular ejection fraction to dynamic exercise is a rise of at least 5\% $\%^{18-20}$ and in patients with coronary heart disease failure to rise implies exercise induced myocardial ischaemia. Antianginal treatment, by preventing the development of ischaemia, might be expected to allow a normal response to exercise. But in our patients there was no improvement in left ventricular ejection fraction on atenolol during dynamic exercise, despite the presence of angina pectoris in only three patients. In normal subjects $\beta$ blockade impairs the ejection fraction response to exercise $^{2122}$ whereas in controlled studies in patients with ischaemic heart disease both attenuation and enhancement have been reported. ${ }^{23-25}$ If left ventricular ejection fraction falls considerably on exercise because of severe ischaemia, prevention of this by $\beta$ blockade would be expected to improve 
the ejection fraction response. But if ischaemia is mild as in our patients, then only a failure of the ejection fraction to rise normally on placebo would be expected, with little change on $\beta$ blockade.

Only on nifedipine and the combination was there a statistically significant rise in exercise left ventricular ejection fraction. On nifedipine this occurred despite the presence of angina in most patients. Possibly the reduction of left ventricular afterload induced by nifedipine allowed a rise in left ventricular ejection fraction despite the presence of myocardial ischaemia. On the combination the rise in left ventricular ejection fraction was associated with an almost complete abolition of angina pectoris which may have been due to the dual effects of afterload reduction induced by nifedipine and the abolition of myocardial ischaemia by atenolol.

In summary, the combination of nifedipine and atenolol was the most effective antianginal treatment. in this group of patients. In addition on the combination, five patients had a normal left ventricular ejection fraction response to dynamic exercise together with the absence of angina pectoris. The combination was well tolerated and in those four patients who experienced a significant fall in left ventricular ejection fraction on the combination there was no clinical or symptomatic evidence of cardiac failure.

We conclude that the combination of atenolol and nifedipine is a safe and effective treatment of angina pectoris and is superior to either atenolol or nifedipine alone in patients with stable exertional angina pectoris. Indeed our data suggest that nifedipine may be used to best advantage in combination with a $\beta$ blocker rather than as a single agent. These results should not be extrapolated to other calcium antagonists or to patients with poor left ventricular function.

I N F was supported by a grant from the British Heart Foundation.

\section{References}

1 Dargie HJ, Lynch PG, Krikler D, Harris L, Krikler S. Nifedipine and propranolol: a beneficial drug interaction. $A m \mathcal{F}$ Med 1981;71:676-82.

2 Tweddel AC, Beattie JM, Murray RG, Hutton I. The combination of nifedipine and propranolol in the management of angina pectoris. $\mathrm{Br} \mathcal{f} \mathrm{Clin}$ Pharmacol 1981;12:229-33.

3 Leon MB, Rosing DR, Bonow RO, Lipson LC, Epstein SE. Clinical efficacy of verapamil alone and combined with propranolol in treating patients with chronic stable angina pectoris. Am $\mathcal{F}$ Cardiol 1981;48:131-9.
4 Krikler DM, Harris MB, Rowland E. Calcium-channel blockers and beta blockers: advantages and disadvantages of combination therapy in chronic stable angina pectoris. Am Heart f 1982;104:702-8.

5 Packer M, Leon MB, Bonow RO, Kieval J, Rosing DR, Bala Subramanian V. Hemodynamic and clinical effects of combined verapamil and propranolol therapy in angina pectoris. Am f Cardiol 1982;50:903-12.

6 Hendry PD. Comparative pharmacology of calcium antagonists: nifedipine, verapamil and diltiazem. $\mathrm{Am} \mathcal{F}$ Cardiol 1980;46:1047-58.

7 Fox K, Selwyn A, Shillingford J. Precordial electrocardiograph mapping after exercise in the diagnosis of coronary artery disease. $\mathrm{Am} \mathcal{F}$ Cardiol 1979;43:541-6.

8 Peto R, Pike MC, Armitage P, et al. Design and analysis of randomised clinical trials requiring prolonged observation of each patient. II Analysis and examples. Br f Cancer 1977;35:1-39.

9 Kurnik PB, Tierfenbrunn AJ, Ludbrook PA. The dependence of the cardiac effects of nifedipine on the responses of the peripheral vascular system. Circulation 1984;69:963-72.

10 Robinson BF, Dobbs RJ, Kelsey CR. Effects of nifedipine on resistance vessels, arteries and veins in man. Br f Clin Pharmacol 1980;10:433-8.

11 Emanuelsson $\mathrm{H}$, Holmberg $\mathrm{S}$. Mechanisms of angina relief after nifedipine: a hemodynamic and myocardial metabolic study. Circulation 1983;68:124-30.

12 Anastassiades CJ. Nifedipine and beta blocker drugs. Br Med f 1980;281:1251-2.

13 Opie LH, White. DA. Adverse interaction between nifedipine and beta blockade. $\mathrm{Br}$ Med f 1980;281:1462.

14 Robson RH, Vishwanath MC. Nifedipine and beta blockade as a cause of cardiac failure. $\mathrm{Br}$ Med $\mathcal{F}$ 1982;284:104.

15 Joshi PI, Dalal JJ, Ruttley MSJ, Sheridan DJ, Henderson AH. Nifedipine and left ventricular function in beta-blocked patients. Br Heart $\mathcal{F}$ 1981;45:457-9.

16 Rowland E, Razis P, Sugrue D, Krikler DM. Acute and chronic haemodynamic and electrophysiological effects of nifedipine in patients receiving atenolol. $\mathrm{Br}$ Heart $\mathcal{f}$ 1983;50:383-9.

17 Johnston DL, Lesoway R, Humen DP, Kostuk WJ. Clinical and hemodynamic evaluation of propranolol in combination with verapamil, nifedipine and diltiazem in exertional angina pectoris: a placebo controlled, double blind, randomised, crossover study. $\mathrm{Am} \mathcal{F}$ Cardiol 1985;55:680-7.

18 Kalff V, Kelly MJ, Jennings GL, et al. The radionuclide assessment of left ventricular function using graded exercise in normal subjects. Aust $N Z \mathcal{F}$ Med 1980;10:533-9.

19 Slutsky R, Karliner J, Ricci D. Response of the left ventricular volume to exercise in man assessed by radionuclide equilibrium angiography. Circulation 1979;60:565-71.

20 Demher GJ, Lewis SE, Hillis LD, Corbett J, Parkey RW, Willerson JT. Exercise-induced alterations in left ventricular volumes and the pressure-volume relationship: a sensitive indicator of left ventricular dysfunction in patients with coronary artery disease. Circulation 1981;63:1008-18. 
21 Marshall RC, Wisenberg G, Schelbert HR, Heinze E. Effect of oral propranolol on rest, exercise and post exercise left ventricular performance in normal subjects and patients with coronary artery disease. Circulation 1981;63:572-83.

22 Port S, Cobb FR, Jones RH. Effects of propranolol on left ventricular function in normal men. Circulation 1980;61:358-66.

23 Johnstone DL, Gebhardt VA, Donald A, Kostuk WJ. Comparative effects of propranolol and yerapamil alone and in combination on left ventricular function and volumes in patients with chronic exertional angina: a double blind, placebo controlled, randomised cross over study with radionuclide ventriculography. Circulation 1983;68:1280-9.

24 Dehmer GJ, Falkoff M, Lewis SE, Hillis LD, Parkey RW, Willerson JT. Effect of oral propranolol on rest and exercise left ventricular ejection fraction, volumes, and segmental wall motion in patients with angina pectoris: assessment with equilibrium gated blood pool imaging. Br Heart $\mathcal{F} 1981 ; 45: 656-66$.

25 Lindsay J Jr, Nolan NG, Goldstein SA, Bacos JM. Effects of beta-adrenergic blocking drugs on sensitivity and specificity of radionuclide ventriculography during exercise in patients with coronary heart disease. $\mathrm{Am}$ Heart $\mathcal{F}$ 1983;106:271-7. 